\title{
Publicar y mirar. La presentación del sí mismo online
}

\section{Checking and posting. The presentation of the self online}

\author{
Magdalena Lemus magdalenalemus.2@gmail.com \\ http://orcid.org/0000-0002-7213-8688 \\ Centro Interdisciplinario de Metodología de las Ciencias Sociales; Instituto de Investigación en \\ Humanidades y Ciencias Sociales; Universidad Nacional de La Plata/ Consejo Nacional de \\ Investigaciones Científicas y Técnicas (Argentina)
}

\section{Resumen}

Este artículo analiza las prácticas de presentación del sí mismo online en Instagram de jóvenes estudiantes secundarios de clases medias altas. En particular, indaga en las dinámicas y lógicas que organizan los modos de exhibir la propia vida y mirar vidas ajenas y se interroga por las posibilidades de exploración con la propia personalidad en los entornos virtuales. La metodología combinó entrevistas biográficas con observaciones virtuales de los perfiles de Instagram de 27 varones y mujeres de entre 15 y 18 años. 
Nuestros hallazgos destacan la existencia de regulaciones grupales que tensionan las posibilidades infinitas de exploración que podría ofrecer la red. A su vez, indican que las prácticas para presentarse en espacios virtuales, se construyen en estrecha relación con lo que ocurre offline. Junto a esto, un conjunto de estereotipos de género redundan en posiciones desventajosas para las jóvenes, tanto dentro como fuera del espacio virtual.

Palabras clave: Jóvenes; redes sociales virtuales; Instagram.

Abstract

Virtual social networks have been highlighted as places to express own ideas and personality freely, as well as to meet people and build relation ships. Taking Goffman's dramaturgical approach, we analyze the presentation of the self online made in Instagram by teenagers from upper middle class. By doing this, we intended to understand the dynamics behind their use of this platform. We developed our methodological approach by combining the biographical method with virtual observations of 27 boys and girls Instagram profiles.

Our main findings suggest that virtual social networks are bounded by powerful rules of behaviour that take place both online and offline. Moreover, within these rules, we found gender inequalities and stereotypes that tend to be reproduced both by boys and girls.

Keywords: Young people; virtual social networks; Instagram.

Perspectivas optimistas respecto de las posibilidades que brinda Internet, han enfatizado su carácter de reserva infinita de contenidos e información, donde las personas pueden acceder a materiales alternativos o inaccesibles (Urresti, 2008). A su vez, ciertos espacios virtuales han sido destacados por su potencial para explorar en la construcción de la propia subjetividad (Turkle, 1997), expresarse y relacionarse con los otros, especialmente en el caso de los jóvenes (Gil-Juárez, Vall-llovera y Feliu, 2010) (1).

Sin embargo, ¿en qué tipo de prácticas se traducen estas posibilidades?, los jóvenes ¿se sienten habilitados a jugar con su propia personalidad online? Este artículo tiene por objetivo comprender las modalidades de presentación del sí mismo en las redes sociales virtuales y su relación con los modos de construcción de las subjetividades juveniles contemporáneas. Para ello, a partir de una combinación de observaciones virtuales y enfoque biográfico, caracterizamos 
las prácticas de presentación del self online de jóvenes de clases medias altas y analizamos los significados que poseen en las tramas de sociabilidad juvenil (2). Nuestros hallazgos ponen en tensión la celebrada potencialidad de Internet y las redes sociales virtuales en términos de "libre expresión", considerándolos como ámbitos de sociabilidad normada, no anárquica ni exenta de condicionantes.

\section{Referencias teóricas}

Los aportes de Goffman (2012) son clave para entender cómo los jóvenes se presentan como individuos y construyen identificaciones. Desde esta perspectiva, la persona se percibe a sí misma a partir de la mirada y la interacción con los otros. El "yo" es resultado de procesos de internalización de estructuras culturales y posiciones sociales, en las que se incorporan reglas y valores, así como expectativas recíprocas para la interacción (Goffman, 2012). El "sí mismo" es un "tipo de imagen, por lo general estimable, que el individuo intenta efectivamente que le atribuyan los demás cuando está en escena y actúa conforme a su personaje" (Goffman, 2012: 282). El autor, diferencia al sí mismo de su poseedor: "el "sí mismo", como un personaje representado, no es algo orgánico que tenga una ubicación específica y cuyo destino fundamental sea nacer, madurar y morir; es un efecto dramático que surge difusamente en la escena representada" (Ibídem: 283).

A su vez, entendemos a las redes sociales virtuales como establecimientos sociales, en tanto espacios con "barreras establecidas para la percepción, en el cual se desarrolla de modo regular un tipo determinado de actividad (...) con un equipo de actuantes que cooperan para presentar al auditorio una definición dada de la situación" (Ibídem: 267).

Por último, el concepto de conveniencia, en tanto "compromiso por medio del cual cada uno, al renunciar a la anarquía de los impulsos individuales, da anticipos a la vida colectiva, con el objeto de retirar sus ganancias simbólicas necesariamente diferidas en el tiempo" (Mayol, 1999: 7) nos ayuda a comprender cómo se organiza la presentación online. De acuerdo con Mayol: "La contrapartida de esta coerción es para el usuario la certeza de ser reconocido, "considerado" por el entorno, y así cimentar en su provecho una relación de fuerzas en las diversas trayectorias que recorre" (1999: 7). 


\section{Estrategia metodológica}

Nuestra investigación se inscribió en el paradigma interpretativo (Vasilachis, 1992) con una orientación no estándar y exploratoria (Marradi, Archenti y Piovani, 2010). A su vez, elegimos la perspectiva biográfica (Muñiz Terra, 2012) por su potencialidad para reconstruir las trayectorias de apropiación de TD de los jóvenes a lo largo de su vida. A partir de esto, buscamos comprender de forma diacrónica, la construcción de vínculos, prácticas y representaciones de las tecnologías digitales (en adelante, TD).

La población de estudio se conformó con varones y mujeres estudiantes secundarios, de clases medias altas de la ciudad de La Plata. El punto de acceso fueron las escuelas a las que asistían (dos de gestión privada sin subvención estatal y una pública dependiente de la Universidad Nacional de La Plata), que seleccionamos a partir del: tipo de gestión, matrícula, cuota, jornada y porcentaje de subvención por parte del Estado y de entrevistas con informantes calificados.

Siguiendo los principios del muestreo intencional (Marradi et al., 2010), definimos los siguientes criterios para la selección de los entrevistados: 1. socioeconómicos (barrio de residencia; cantidad de personas y habitaciones en el hogar; propiedad del lugar de residencia; formación educativa y situación laboral de padre y madre); 2. tecnológicos (cantidad, diversidad y antigüedad de TD en el hogar; intensidad y usos frecuentes de computadora, celular e internet; participación en redes sociales y en plataformas de juegos); y 3. personales (género; intereses y hobbies en el tiempo libre; realización de viajes al exterior; proyectos a futuro; estancias en instituciones educativas en países extranjeros; realización de cursos intensivos de idioma en el extranjero: viajes a países angloparlantes y a Europa).

La cantidad de entrevistados se determinó por saturación teórica (Glaser y Strauss, 1967). Seleccionamos 27 jóvenes, entre varones y mujeres, que se encontraban cursando el ciclo orientado de la escuela secundaria (cuarto a sexto año), con quienes realizamos 51 entrevistas biográficas. Además, hicimos observaciones en sus perfiles de Instagram y visitamos sus páginas web preferidas. Dentro de las TD, nos concentramos en estudiar la apropiación de internet, smartphones, consolas de videojuegos y computadoras (tablets, netbooks, notebooks y de computadoras de escritorio).

\section{De publicar sin filtro a la minuciosa edición del sí mismo}

La articulación entre momentos biográficos y el desarrollo de TD, ha dado lugar a formas de 
presentación del sí mismo online con distintas características y lógicas a lo largo del tiempo. A partir del análisis de las historias de vida de los entrevistados, hallamos que sus vínculos con las TD se destacan por la abundancia y diversidad de dispositivos tecnológicos presentes en el entorno (hogar y escuela), así como su disponibilidad desde temprana edad y un acceso ininterrumpido a estos bienes.

Padres y madres con amplios saberes para operar computadoras e Internet, con un uso frecuente y fluido de éstos, actuaron a modo de iniciadores de las trayectorias de apropiación. Más tarde, hermanos mayores y amigos emergieron otros actores con mayor centralidad. Mientras los adultos enseñaron cuestiones generales para el uso de la computadora e internet, con los pares se construyeron instancias de aprendizaje basadas en el intercambio y la reciprocidad (Ito, Baumer, Bittanti, boyd, Cody, Herr-Stephenson, 2010) orientadas al desarrollo de habilidades específicas, como el uso de redes sociales virtuales y juegos online.

Los recorridos de los entrevistados con las TD fueron lineales y homogéneos. En primer lugar, siguieron un mismo patrón (en temporalidades, prácticas y dispositivos) de incorporación de artefactos y software a su vida cotidiana, así como de migración hacia otras TD (Caron y Caronia, 2007), o reemplazo de dispositivos y aplicaciones. En segundo lugar, la homogeneidad se explica por la existencia de condiciones similares para la apropiación, en todos los casos analizados. El acceso a TD, los saberes disponibles en el hogar y las habilidades desarrolladas a partir de la apropiación, tienen semejanzas entre varones y mujeres, sin distinción del colegio de procedencia. Los usos y las representaciones sobre las TD que circulan entre éstos jóvenes, son también afines.

En los primeros usos de las TD, no tenían lugar las acciones de presentación del sí mismo. Los entrevistados usaban computadoras para entretenerse con juegos que ya estaban instalados, se cargaban en las consolas de videojuegos o estaban disponibles en Internet, pero para los cuales no se requerían, ni tampoco se habilitaban, prácticas de presentación del sí mismo.

La construcción de perfiles virtuales que excedieran la elección de un avatar y un alias (como ocurría en servicios de mensajería virtual), surgieron con Facebook, espacio que por su diseño requería que los usuarios volcaran información personal. Durante la niñez (3), el Facebook fue usado como una plataforma para jugar al "Pet Society". Para el inicio de la secundaria, lo que habilitaba técnicamente Facebook, comenzó a ser procesado por los jóvenes, especialmente por las mujeres, como la posibilidad de realizar "books" de fotos con las amigas, imitando las prácticas de las modelos, posando, a veces maquilladas y vestidas para la ocasión.

Si bien hacer books no estaba tan extendido entre los varones, sí era frecuente registrar con cámaras digitales las reuniones con amigos y subir esas fotos a Facebook. Para ambos géneros, esta etapa se caracterizó por un uso intensivo de esta red, en la que buscaban 
"publicar todo", "todo el tiempo" y "sin filtros", fueran fotos, videos o comentarios. Luego, de "publicar todo" pasaron a posteos más esporádicos (una vez por semana, por ejemplo) de fotos elegidas con mayor detenimiento, buscando las que favorecían la imagen individual y mostraban situaciones consideradas interesantes y divertidas (cumpleaños, salidas y viajes).

Las redes sociales virtuales son parte de un "ecosistema de medios conectivos" (van Dijck, 2016: 43), de una estructura mayor, dentro del cual las TD se influyen mutuamente y cada cambio en una plataforma o aplicación tiene repercusiones en las demás (van Dijck, 2016). Al analizar las trayectorias de apropiación de las TD, hallamos que las prácticas de presentación del sí mismo online se transformaron a lo largo del recorrido biográfico, en vinculación con cambios técnicos en los dispositivos y en los espacios virtuales, así como con formas sociales de vivir la niñez, la preadolescencia y la juventud para las clases medias altas. La aparición de adultos (padres, madres y, especialmente, abuelos) en redes como Facebook, junto con el surgimiento de nuevas plataformas virtuales, contribuyó a que los jóvenes migraran hacia nuevos espacios y con ello cambió también el modo de moverse allí. La universalización de Instagram en los ámbitos de sociabilidad de los jóvenes reformuló los códigos en torno a la privacidad y el uso de hashtags para ganar visibilidad. Mientras fue usada por pocas personas dentro de los círculos más cercanos, la privacidad quedó subsumida a la búsqueda de visibilidad que llegaba en forma de "me gustas" y nuevos seguidores. Cuando ese espacio social se volvió masivo, se reformularon los modos de generar adhesión y la privacidad.

A su vez, cuando una red social virtual es nueva en los ámbitos de sociabilidad, se da un tipo de uso caracterizado por "la emoción" y "el vicio" que implica estar conectado muchas horas por día mirando las actividad de los contactos y publicando frecuentemente (varias veces al día) y "sin filtro". Nombrar a esta práctica como "sin filtro" no implica que no se despliegue una performance en el sentido dado por Goffman (2012). Por el contrario, al caracterizarla así los jóvenes establecen una línea divisoria entre las modalidades de presentación pasadas y actuales, en las que se da a entender que, para la presentación del sí mismo contemporánea, hay una búsqueda explícita por exhibir un contenido cuidadosamente seleccionado y estilizado, filtrado.

Pasado este primer momento de "emoción", deviene una estabilización de los significados y prácticas que tienen lugar en cada espacio virtual. Por ejemplo, cuando aún pocas personas del entorno de los entrevistados utilizaban Instagram, el hashtag junto con la configuración de sus perfiles como públicos y la publicación de fotos varias veces a la semana, eran tácticas que los jóvenes desplegaban para ganar visibilidad. En la actualidad, con Instagram extendido en los ámbitos de sociabilidad de los jóvenes de clases medias altas platenses, el uso de hashtag cayó en desuso y es considerado como "descanso" (4). Es decir, quien lo usa ahora no 
comprendió el código: el hashtag era significativo en un momento de menor popularidad de la red y de sus usuarios:

Antes subíamos fotos ponele y poníamos hashtags para tener más me gusta (...) el tema es que ahora no se usan, como que no podés poner los hashtags. Al principio éramos pocos los que teníamos Instagram (...) a medida que se fue sumando más gente era más normal tener 11 "me gustas", porque como que todo mi círculo tiene Instagram, me sigue, me pone "me gusta", entonces ya no necesito que el otro "x" me ponga "me gusta" (Valentina, 17 años, comunicación personal).

El relato de Valentina ilustra la transformación del uso de Instagram, con el pasaje de la publicación frecuente "de todo", a una detallada selección del contenido a mostrar y de las personas con las que relacionarse. Mientras que en la preadolescencia "no importaba nada" sobre el contenido que se publicaba en Facebook porque lo significativo era publicar, llegada la juventud, el juicio y la mirada de los otros significativos (de Singly, 2005), pertenecientes a los círculos de sociabilidad (colegios, clubes, gimnasios, etcétera) y ajenos a la familia, empezó a ganar protagonismo. En este sentido, la presentación en las redes sociales virtuales comenzó a regirse con mayor fuerza por las expectativas recíprocas (Goffman, 2012) de los jóvenes respecto a esa instancia de exhibición e interacción con sus pares. De esta manera, si de acuerdo con Goffman (2012), la existencia de este tipo de expectativas era un elemento central en las diversas interacciones sociales offline, en nuestro caso también son elementos clave que operan en el modo en que los jóvenes eligen mostrarse en las redes sociales virtuales y, a su vez, evalúan las formas en que se presentan los otros.

Junto a esto, entre la preadolescencia y la juventud, en un momento de pronunciadas transformaciones en la fisonomía y de construcción de la propia imagen corporal, los discursos acerca de un "deber ser" de género comienzan a adquirir entre los jóvenes, y especialmente entre las mujeres, gran importancia. El uso de las redes por parte de famosos (cantantes, modelos, actrices/actores, deportistas) y la explotación comercial de estos espacios vía publicidad, contribuyen a generar estereotipos online de las vidas y cuerpos que son "mostrables" y, por lo tanto, "no mostrables". Así, el pasaje de ser niña a mujer no solo se vive física y psíquicamente offline, sino que también se inscribe en los usos de las redes sociales virtuales a través de prácticas prescriptas y adecuadas, recaudos a tomar sobre la manera de mostrarse, señalados en los discursos de adultos, medios de comunicación y pares.

Por último, las transformaciones técnicas que operan en el tipo de redes sociales virtuales, es decir, lo que el espacio por su diseño permite realizar en cada momento, interviene también en 
cómo los jóvenes se presentan, habilitando y condicionando ciertas formas de ser, ciertos cuerpos (Sibilia, 2013). Para los entrevistados, la presentación del sí mismo online es una actuación ensayada y planificada en la que no queda demasiado margen para la espontaneidad. Estas acciones se inscriben en la tendencia a publicar en Instagram contenidos que contribuyan a una auto-promoción consciente (van Dijck, 2013), debido a la combinación de las características técnicas de la plataforma, que promueve la exhibición de contenidos visuales, y la creencia compartida entre los ámbitos de sociabilidad acerca del tipo de contenidos "más adecuados" para ser publicados en cada espacio virtual (Waterloo, Baumgartner, Jochen y Valkenburg, 2017).

A su vez, las expectativas recíprocas (Goffman, 2012), contribuyen a estructurar las prácticas de presentación del sí mismo online. El contenido que los jóvenes publican, a la vez que los constituye, forma parte de la búsqueda de reconocimiento, valoración y adhesión por parte del otro. Así, el mostrarse en las redes sociales virtuales, es siempre para un otro, en nuestro caso, para un otro cercano y similar, de quien esperan reconocimiento y aprobación, que llegan en forma de comentarios positivos dentro y fuera de las redes sociales virtuales, así como de "me gusta" y de nuevos seguidores.

\section{Exhibición y expresión}

Diversas investigaciones que han de expresarse, mostrarse y exhibirse aparecen regularmente como sinónimos en la literatura, que destaca el potencial de las redes sociales virtuales (GilJuárez et al., 2013; Sibilia, 2013). Sin embargo, entre los entrevistados encontramos una acentuada diferencia entre expresarse y mostrarse, sustentada principalmente en cuestiones de cercanía e intimidad con los contactos, amigos o seguidores en las redes sociales virtuales, así como en las habilitaciones técnicas de cada espacio virtual.

\section{E: ¿Creés que Snapchat o Instagram te permiten expresarte?}

A: Más que expresarte te permiten mostrarte. $Y$, digamos, para expresarte tenés que poner en Twitter redactás en 140 caracteres. Bueno, en WhatsApp no tenés ningún límite y podés decir lo que querés. Pero, bueno, lo que tiene ahí es que vos se lo mandás a un contacto o a un grupo, ¿entendés? Vos sabés a quién se lo estás mandando. En Twitter por ahí no. En Twitter vos lo publicaste y ahí lo ve el que te sigue. (...) es mucho más masivo que el WhatsApp. Y Snapchat e Instagram te permiten más mostrarte, porque lo que hacés en Instagram es subir una foto y a lo sumo le pondrás un pie de foto y nada más. (...) Y la gente no sube cualquier foto. Sube una foto en la que salió bien (Ares, 17 años, 
comunicación personal).

La expresión es considerada una acción que tiene lugar con amigos, parejas, familia, es decir, con quienes se tiene mayor intimidad y cercanía. Si bien no se acota a los ámbitos offline, cuando ocurre en instancias virtuales tiene lugar a través de servicios de mensajería o chats privados y no es objeto de exhibición.

A su vez, las maneras de moverse en las redes sociales virtuales y, por lo tanto, qué mostrar, cómo y cuándo, también se han transformado de manera vertiginosa en las últimas décadas, y todo indica que van a seguir en movimiento (van Dijck, 2016). En la actualidad "Los patrones de comportamiento existente en la socialidad offline (física) se mezclan cada vez más con las normas sociales y sociotécnicas generadas en el entorno online, que adquieren así una nueva dimensionalidad" (van Dijck, 2016: 40). Así, la transformación de las reglas de socialidad, es un proceso gradual de "normalización" (Ídem), por medio del cual los usuarios no solo se van acostumbrando a las nuevas características de las plataformas, entre éstas, a las referentes a la privacidad y la exhibición, sino especialmente a la monetización de los sitios (van Dijck, 2016). Los jóvenes establecen su propia diferenciación entre lo que es publicable y publicitable, y lo que es íntimo y privado. Estas formas de entender la distinción entre lo público y lo privado difieren significativamente de la de los adultos y, en ocasiones, es materia de conversación y tensión en el hogar y la escuela.

La posibilidad de expresión a través de las redes sociales virtuales es así contestada en las opiniones y experiencias de los jóvenes entrevistados:

\begin{abstract}
Soy de esas personas que es como que me acuerdo mucho cuando la gente dice "odio el color blanco", y al otro día viste tiene todo blanco. (...) Y no me gusta a mí tipo ser así (...) si lo decís hablando tal vez es como que la podés pilotear más, si después cambiás de opinión. Pero ya cuando lo publicás. O sea, entiendo que cambies de opinión, pero si no tenés la opinión tan fija yo no la publicaría. Por eso en las redes sociales yo no hablo de política (Gina, 17 años, comunicación personal).
\end{abstract}

Los jóvenes consideran a los espacios virtuales como ámbitos en donde las discusiones suelen ser estériles porque no tiene lugar un "verdadero diálogo" ni "nadie va a cambiar de parecer", al mismo tiempo que este tipo de entorno suele prestarse más para "polémicas" que para "discusiones serias". Además, temen quedar "pegados" a una posición que no saben si van a sostener en el tiempo. La política, como ocurre con Gina, es uno de los tópicos que más reticencia genera en los jóvenes en relación a las redes sociales virtuales. Por un lado, porque estos ámbitos son principalmente concebidos como espacios para mostrarse e interactuar, y no 
como una arena por la que pueda tener cauce la discusión o el conflicto, como ocurre con Twitter (Waterloo et al., 2017). Por el otro, tienen presente que las publicaciones en la web no desaparecen, quedan fijadas en tiempo y espacio y operan a modo de archivo con el cual cotejar posicionamientos. Este tipo de precauciones y temores, contribuyen a entender la significación que para los jóvenes adquieren las presentaciones del sí mismo online: no son prácticas que se realizan livianamente, escindidas de las no virtuales, sino acciones que pueden tener implicancias y consecuencias a largo plazo.

\section{Habilitaciones para presentarse online}

Para DeNora (2000) los dispositivos tecnológicos habilitan a las personas a hacer, a sentir, a la vez que también restringen ciertos cursos de acción (DeNora, 2000). En la misma sintonía, Silverstone (2004) ha propuesto entender a las TD como habilitantes o inhabilitantes, en contraposición a miradas deterministas de las tecnologías. De esta forma, es necesario indagar, en cada caso y en cada contexto, qué prácticas sobre sí mismo y los otros se vuelven posibles al usar las TD. En este sentido, para Fortier y Burkell (2016), las acciones de los jóvenes en las redes sociales virtuales tienen finalidades diversas, a la vez que sostienen distintas expectativas sobre la posibilidad de exhibir y controlar contenidos en cada espacio. En nuestra investigación, hallamos que la participación en las redes sociales virtuales, se desenvuelve a través de dos perfiles predominantes: el "usuario publicador" y el "usuario observador".

El "usuario publicador" postea frecuentemente fotos y videos de las actividades que realiza en su rutina cotidiana (ir al gimnasio, estudiar, estar con amigos) y durante el fin de semana (principalmente salir a la noche, juntarse a la tarde con amigos o familiares, ir a recitales). A su vez, eventos como fiestas de egresados o viajes (al extranjero especialmente) son siempre mostrados y relatados a través de Instagram, en donde adquiere un lugar clave el cuidado estético del contenido que se publica, ya sea de la propia imagen, de paisajes o bienes que consume. Dentro de este grupo, están los "controladores de la imagen" [Image controllers], para quienes el alcance del contenido que publican puede extenderse un poco más allá del núcleo de amigos y familiares más cercanos, pero sin perder el control de quiénes lo siguen y acceden a su perfil online (Fortier y Burkell, 2016: 14). El usuario publicador puede también desenvolverse como un "exhibidor relajado" [Relaxed displayers] (5), que está poco preocupado por la privacidad y considera que el contenido que publica es justamente para ser compartido, no siendo las redes sociales virtuales espacios para mostrar cuestiones privadas o 
íntimas (Fortier y Burkell, 2016: 14).

Por su parte, el "usuario observador", tiene una participación en las redes sociales virtuales más acotada en términos de exhibición, publica de manera poco frecuente y prefiere mostrar fotos de paisajes o actividades con amigos, y no de sí mismo.

En ambos casos, hallamos que sentirse habilitado para publicar o mostrar en las redes sociales virtuales está vinculado con la percepción de sí mismo en el terreno no virtual, es decir, en el contexto más amplio en que se emplaza la presentación del self. En contraposición de lo que podría esperarse respecto al potencial de los espacios virtuales para las personas se sientan más cómodas y libres para expresarse y mostrarse, al analizar conjuntamente entrevistas biográficas con observaciones online y offline, encontramos que las prácticas online tienden a replicar las posiciones subjetivas y prácticas offline en el espacio virtual. Así, en general, quienes se consideraban personas un tanto introvertidas o reservadas, se desenvuelven principalmente como "usuarios observadores":

\begin{abstract}
Yo miro todo, pero yo no soy de la que va a compartir algo, a publicar. Pongo me gusta, por ejemplo en Facebook hay gente que por ahí ve un video que le gusta y lo comparte, yo jamás hago eso. Yo lo miro, a lo sumo, si me gusta mucho, me lo guardo y se lo muestro después a una amiga, o le mando la URL, o la comento para que la vea. Por ahí como que soy medio tímida y no salgo como para que todo el mundo diga "ay, mirá lo que Emilia está diciendo" (Emilia, 17 años, comunicación personal).
\end{abstract}

Las palabras de Emilia ilustran la articulación entre las posibilidades técnicas de las TD con la habilitación subjetiva para presentarse en los espacios virtuales (publicar contenidos, mostrarse). En el caso de los jóvenes de clases medias altas que han crecido en condiciones privilegiadas de acceso a las TD, esta habilitación subjetiva no arraiga en condiciones materiales, sino en las tramas de sociabilidad de las que forman parte y en las percepciones de sí mismos. En este sentido, la presentación del sí mismo online es experimentada de forma desigual por los jóvenes, redundando en que algunos se sienten más "habilitados" para mostrarse que otros. Sin duda, esta habilitación subjetiva no es ajena a las regulaciones en torno a qué tipo de cuerpos y vidas son mostrables.

\title{
Desigualdades de género en la presentación del sí mismo online
}

A lo largo de nuestra investigación fue tomando fuerza el siguiente argumento: buena parte de 
las ideas presentes entre los jóvenes sobre cómo presentarse en las redes sociales virtuales está dirigida principalmente hacia el comportamiento de las mujeres se erige sobre estereotipos de género arraigados en la sociedad argentina contemporánea y redundan en desigualdades para las mujeres. En los relatos de los jóvenes, coexisten y se solapan diversos estereotipos y posturas, con matices y contradicciones.

\section{"No debería no importarme, pero la verdad es que no me importa"}

Un conjunto de prescripciones sobre cómo y cuánto de los cuerpos es "adecuado" mostrar online, recaen entre varones y mujeres, aunque tienen como principales destinatarias a estas últimas. Las respuestas de las jóvenes son diversas, mientras algunas tratan de regirse por éstas prescripciones, otras tienden a desafiarlas un poco más:

Para mí hay un prejuicio. Generalmente con todo, pero en las fotos yo creo que se nota más. Yo creo que ver una foto de un chico en la playa en cuero no tiene la misma repercusión que ver una chica sacándose una foto en malla y subiéndola. Para el caso de la mujer como que siempre lo repudian un poco, como que no está muy bien. (...) Y para el hombre como que es normal, está en la playa, se sacó una foto y listo. No es que lo anda pensando mucho (Pía, 17 años, comunicación personal).

En sus palabras, Pía da cuenta del doble estándar con que se consideran las prácticas online de hombres y mujeres. Esto implica, para las mujeres, una tarea extra al momento de presentarse online que, a la vez, conlleva el trabajo previo offline de evaluar la propia vestimenta y actitud. También pone de relieve la ambivalencia de esta experiencia: a la vez que es consciente de las reglas y del mayor peso con la que éstas recaen sobre su género, busca ponerlas en tensión y trata de "que no le importen". Su posicionamiento trasciende el relato y se plasma, como vimos en las observaciones virtuales, en los modos en que ella se muestra en Instagram: tiene el perfil abierto y publica con frecuencia fotos y videos en donde se la ve sola o acompañada, en diversas actividades, como haciendo crossfit, desfilando en un evento, posando en bikini en la playa o en su casa antes de salir a bailar, mostrando el look elegido y su cuerpo modelado en el gimnasio. Como nos comentaba:

Te digo la verdad... no debería no importarme, pero la verdad es que no me importa. Depende la persona. Yo sé que, si una chica sube una foto en malla, a no ser que la foto sea desubicada, no digo nada. Listo, fue a la playa y se sacó una foto y ya está, y lo mismo 
digo del hombre. Pero hay personas que yo sé que no son así (Pía, 17 años, comunicación personal).

Si bien Pía se considera más desprejuiciada para mostrar su cuerpo que otras jóvenes de su edad y clase social, también en su discurso hay ciertos límites para la exhibición: "que la foto sea desubicada". Así, aunque cada persona puede mostrarse cómo desee en los espacios virtuales, hay ciertos límites que deberían reconocerse, por ejemplo "una pose o que no se le vea la cara y se saque nada más el cuerpo" (Pía, 17 años, comunicación personal). Sin embargo, estos límites deberían ser equitativos para varones y mujeres:

Siempre se la vincula a la mujer como que es la que tiene la culpa, que se saca una foto así y ... "no tenés que sacarte porque sos mujer", y al hombre nunca se le dice nada. Y la verdad es esa, tendrían que poner en una igualdad esas cosas. De los hombres como que nunca van a decir nada, porque van a pensar que es normal. Y las mujeres [les van a decir]: “ ¿cómo le van a mandar un video a un flaco que no conoce, que no está de novio, que no tiene confianza, sabiendo lo que le podría pasar? (Pía, 17 años, comunicación personal).

En sus palabras, Pía pone de manifiesto su disconformidad respecto a los discursos dominantes sobre cómo deben mostrarse las mujeres en las redes sociales virtuales, y destaca la posición desventajosa respecto de los varones. A su vez, considera que es injusto que sean las mujeres quienes tengan que desarrollar estrategias de resguardo diferenciales.

\section{"Si vos sabés más o menos en lo que te estás metiendo tendrías que tener cuidado"}

Si la posición esgrimida por Pía ponía el acento principalmente en la posibilidad de mostrarse cómo persona desee y que, en todo caso, los juicios y recaudos recaigan de manera más igualitaria sobre varones y mujeres, las posturas de otras jóvenes, tienden a reforzar los estereotipos de género existentes:

Si vos sabés más o menos en lo que te estás metiendo tendrías que tener un cuidado. $O$ sea, hay formas de prevenir digamos. Una chica que tiene unas súper tetas y anda siempre con un escote abierto, por más que la chica sea buenísima y todo, la gente va a hablar más de eso que de otra que está con el escote cerrado. Entonces si vos no te querés meter en eso, tenés que tener un poco más de cuidado(Emilia, 17 años, comunicación personal).

Si bien Emilia da cuenta de la existencia de evaluaciones diferenciales sobre los 
comportamientos "adecuados" para hombres y mujeres, su posición termina reproduciendo las desigualdades existentes al enfatizar la responsabilidad exclusiva de las mujeres al momento de exhibirse y, así, delegar en ellas el cuidado y protección frente a los otros. Así, en este discurso se ponen en acción dos cuestiones: la importancia de mostrarse de una forma "adecuada" en esos espacios, y las estigmatizaciones, miradas condenatorias o el peligro de ataque físico, como consecuencias posibles de una "inadecuada" exhibición online. La exhibición del cuerpo también aparece asociada al modo de circulación de la información en la web:

Para mí no da que te estés mostrando con Instagram. Igual podés hacerlo, es tu vida pero yo no lo haría porque me parece que no da andar mostrándote así a desconocidos. Si lo tenés público lo ve cualquier persona y no da (...) No es que es solo tuyo. No es que sacás una foto y queda en la cámara. Está en internet, está en cualquier lado. (...) la mujer como que está todo eso de que puede ser violada, cosas así. El varón no tiene todo ese peso (Violeta, 17 años, comunicación personal).

Aunque Violeta reconoce la existencia de peligros basados en el género, la responsabilidad del cuidado recae en la mujer. A su vez, enfatiza el carácter incontrolable de la información una vez que es publicada en la web, cuestión que se suma a los factores a tener en cuenta al mostrarse.

Como pudimos analizar a partir de las observaciones virtuales, las posturas discursivas de éstas jóvenes respecto a la exhibición online son acompañadas de una participación en Instagram a modo de "usuarias fantasmas", tal como ellas lo definieron, las "usuarias observadoras" que definimos en el apartado anterior. Es decir, tienen cuentas activas, siguen a personas y dan "me gusta", pero no tienen ninguna foto publicada, salvo la de perfil, en donde ambas están con amigas y se ven únicamente sus rostros. Esta forma de habitar Instagram debe entenderse a partir de la triple articulación de los miedos y peligros antes señalados, la búsqueda por no exhibir "demasiado" y con la imposibilidad, según sus propios términos, de no cumplir con el estándar establecido en relación a qué cuerpos y vidas son mostrables.

\section{"A la mujer la juzgan mucho más"}

Entre los varones encontramos discursos que dan cuenta de los juicios diferenciales a los que son sometidos hombres y mujeres, al momento de mostrarse dentro y fuera de las redes, y en donde a éstas se les adjudican posiciones y caracterizaciones desventajosas. En relación con 
las opiniones de los varones entrevistados, debemos tener en cuenta algunas cuestiones. En primer lugar, la existencia, a nivel macro social, de un proceso de activación masiva de las discusiones sobre violencia de género que ganó estado mediático en 2015 con el surgimiento de "Ni Una Menos". En segundo lugar, no podemos obviar que los posicionamientos de los varones fueron desarrollados en un contexto de entrevista privada e individual con una mujer investigadora. Así, si bien no podemos atribuir las respuestas de los jóvenes al peso que puede haber tenido en ellos "la mirada de la socióloga", tampoco podemos soslayar esta cuestión. Sobre la exhibición online del cuerpo, Ares nos comentaba:
E: ¿Pero creés que hay prejuicios o cierta mirada si eso lo hace una mujer? ¿Es distinto que si lo hace un hombre?
A: ¡Sí, sí!. Digamos, hablando mal y pronto, si una mujer lo hace es una puta y si un hombre lo hace es un fachero. A ver, si un varón sube por ahí una foto ponele en la playa, con una malla, digamos, y medio en cuero, no se le dice nada. $Y$ por ahí si una mina sube una foto por ahí más jugada se le dice algo. Pero ya si un varón sube una foto en bolas también se le dice. No es que no, se le dice algo (Ares, 17 años, comunicación personal).

Es interesante notar que Ares menciona ciertos límites que pareciera que los varones tampoco pueden cruzar, al señalar que a ellos también se les marca cuando suben "fotos en bolas". Otro de los jóvenes, señala dos elementos más a considerar junto con el juicio diferencial que recae sobre las mujeres al momento de presentarse online:

\begin{abstract}
O sea, a la mujer la juzgan mucho más. Es como que tiene el doble de miradas encima, porque, de última, al hombre no le dicen nada, pero a la mujer sí. Por ahí se siente más presionada. Se comenta después. Ese es el problema, que a un varón nadie le dice nada (Marcos, 17 años, comunicación personal).
\end{abstract}

La imposibilidad de controlar la forma y velocidad con que se difunde la información por Internet, es un aspecto visualizado por los jóvenes como una condición que impone Internet y que hay que tener en cuenta para moverse allí. Además, existen "presiones extras" que reciben las mujeres para presentarse en las redes sociales virtuales: tienen que ostentar cuerpos ajustados a los cánones de belleza dominantes pero que deben ser exhibidos "solo hasta cierto punto". Así, "[las mujeres] tienen doble presión con las redes sociales. Ellas se quieren mostrar lindas y todo, y dar buena impresión" (Marcos, 17 años, comunicación personal). En las palabras de Marcos, "dar buena impresión" resume el esfuerzo de las jóvenes por adaptar la exhibición a los límites de "lo adecuado" para estos establecimientos sociales. 
En este contexto, tanto varones como mujeres sienten que son evaluados cuando muestran sus cuerpos en las redes. Sin embargo, los calificativos son desiguales: trolas, putas vs. bananas, giles o facheros y las adjetivaciones que reciben las mujeres, aparecen reñidas con las moralidades dominantes tanto online como offline. A su vez, los estigmas que recaen sobre ellas cuando no cumplen con los códigos del mostrarse, actúan a modo de justificante no solo de agresiones o habladurías virtuales, sino también como elementos que pueden contribuir a abusos y maltratos offline.

\section{Conclusiones}

A lo largo del artículo analizamos las características que adquiere la presentación del self en espacios virtuales. Hallamos que hay un esfuerzo por parte de los jóvenes para volver a sus cuerpos y vidas cotidianas objeto de publicación, el cual debe ser entendido en el marco del proceso de estilización y espectacularización de la vida cotidiana (Sibilia, 2013). Junto a esto, hay entre los jóvenes un profundo conocimiento de los establecimientos sociales (Goffman, 2012) en que se desenvuelven, de las lógicas que gobiernan estos espacios, así como de las expectativas de los otros sobre la propia conducta. En este sentido, las expectativas recíprocas (Goffman, 2012) acerca de qué es esperable en la presentación online, juegan un rol clave en la construcción de perfiles en las redes sociales virtuales.

A su vez, a contramano de los pronósticos que enfatizan la tendencia a la individualización y fragmentación de las prácticas y experiencias online, encontramos prácticas orientadas a la construcción y mantenimiento de códigos y normas compartidos. Así, la de presentación del sí mismo online se emplaza sobre normatividades y desigualdades de género offline comunes a los ámbitos de sociabilidad juvenil. De acuerdo con Winocur, a la vez que las TD habilitan a los individuos a entrar en contacto con mundos desconocidos y a traspasar fronteras de distinto tipo, "paradójicamente tendemos a desarrollar mayores dispositivos simbólicos del orden familiar y local para interpretarlos" (Winocur, 2013: 258). Lejos de erosionarse las normas de interacción existentes en la arena offline, hay una construcción de regulaciones para moverse en el terreno virtual que guarda una estrecha relación con lo no virtual. De esta forma, hay prácticas que se habilitan en la virtualidad, que pueden dar lugar a la exploración, a la constante edición de la manera en que las personas se presentan en sociedad, pero también hay una construcción de reglas de interacción propias de esos espacios. Los jóvenes se mueven online en un doble juego que se desenvuelve entre la exhibición de los propios gustos, intereses y prácticas y el amoldamiento a criterios colectivamente validados para realizar esa 
presentación.

Por último, encontramos un acentuado proceso de reproducción online de los estereotipos de género dominantes en nuestra sociedad. Esta reproducción de cánones de belleza y comportamientos hegemónicos afecta de manera desigual a los jóvenes, recayendo el mayor peso sobre las mujeres. Así, frente a las aparentemente ilimitadas formas de ser, estar y explorar con uno mismo en los espacios virtuales, hallamos modalidades de reconfiguración online de las desigualdades de género ya existentes.

\section{Notas}

(1) Sin desconocer las discusiones en torno al lenguaje inclusivo, con el objetivo de agilizar la presentación y lectura, en este trabajo utilizamos el masculino para referirnos a la totalidad de las personas entrevistadas y realizamos distinciones de géneros únicamente en los pasajes en los que resulta significativo y relevante para el desarrollo de los argumentos.

(2) Este trabajo es producto de una tesis doctoral que abordó las relaciones entre apropiación de TD y configuración de desigualdades, desde la perspectiva de los actores privilegiados en las relaciones de desigualdad. Uno de los hallazgos de la tesis, es la identificación de una estrecha relación entre la construcción de subjetividad online y offline y las prácticas de enclasamiento, cuestión que damos por supuesta a lo largo de este artículo.

(3) Analíticamente, identificamos tres momentos: 1. niñez, hasta 10 años; 2. preadolescencia, de 10 a 13 años; y 3. juventud, desde los 13 años.

(4) "Descanso" hace referencia a la categoría nativa que, de forma burlona, alude a quien no entiende o desconoce los códigos de interacción.

(5) "Relajado" refiere únicamente a la privacidad y el alcance de las publicaciones, y no a la dedicación puesta a generar y editar el contenido que se exhibe.

\section{Bibliografía}

Caron, A. H. y Caronia, L. (2007). Moving cultures. Mobile Communication in Everyday Life. Montreal: McGill Queen's University Press.

DeNora, T. (2000). Music en Everyday Life. Cambrigde: Cambridge University Press.

de Singly, F. (2005). How to put an end or not to youth. Youth studies magazine, 71, pp. 107117. 
Fortier, A. y Burkell, J. (2016). Display and control in online social spaces: Towards a typology of users. New media \& society, 20(3), 845-861.

Gil-Juárez, A.; Vall-llovera, M. y Feliu, J. (2010). Consumo de TIC y Subjetividades Emergentes: ¿Problemas nuevos? Intervención Psicosocial, 19(1), 19-26.

Glaser, B. y Strauss, A. (1967). The discovery of grounded theory. Chicago: Aldine.

Goffman, E. (2012). La presentación de la persona en la vida cotidiana. Buenos Aires. Amorrortu.

Ito, M.; Baumer, S.; Bittanti, M.; boyd, d.; Cody, R.; Herr-Stephenson, B.; Horst, H. A.; Lange, P. G.; Mahendran, D.; Martínez, K. Z.; Pascoe, C.J.; Perkel, D.; Robinson, L.; Sims, C. y Tripp, L. (2010). Hanging out, messing around, and geeking out. kids living and learning with New Media. Cambridge/Massachusetts: MIT Press.

Marradi, A.; Archenti, N. y Piovani, J. I. (2010). Metodología de las Ciencias Sociales. Buenos Aires: Cengage Learning Argentina.

Mayol, P. (1999). El barrio. En De Certeau, M.; Luce, G. y Mayol, P. La invención de lo cotidiano. Habitar, cocinar (Tomo 2) (pp. 5-12). México DF: Universidad Iberoamericana, Instituto Tecnológico y de Estudios Superiores de Occidente.

Muñiz Terra, L. (2012). Carreras y trayectorias laborales: una revisión crítica de las principales aproximaciones teórico-metodológicas para su abordaje. ReIMeCS, 2(1), 36-65.

Sibilia, P. (2013). La intimidad como espectáculo. Buenos Aires: Fondo de Cultura Económica.

Silverstone, R. (2004). ¿Por qué estudiar los medios? Buenos Aires: Amorrortu Editores.

Turkle, S. (1997). La vida en la pantalla. Barcelona: Paidós Ibérica.

Urresti, M. (2008). Ciberculturas juveniles. Los jóvenes, sus prácticas y representaciones en la era de Internet. Buenos Aires: La Crujía.

van Dijck, J. (2013). 'You have one identity': performing the self on Facebook and Linkedln. Media Culture Society, 35(2), 199-215.

van Dijck, J. (2016). La cultura de la conectividad: una historia crítica de las redes sociales. Buenos Aires: Siglo Veintiuno Editores.

Vasilachis, I. (1992). Métodos Cualitativos I. Los problemas teórico-epistemológicos. Buenos Aires: Centro Editor de América Latina.

Waterloo, S. F.; Baumgartner, S. E.; Jochen, P. y Valkenburg, P. M. (2017). Norms of online expressions of emotion: Comparing Facebook, Twitter, Instagram, and WhatsApp. New media \& society, 20(5), 1813-1831.

Winocur, R. (2013). Los diversos digitales y mediáticos que nos habitan cotidianamente. Hegemonía cultural y políticas de la diferencia. Buenos Aires: Consejo Latinoamericano de Ciencias Sociales. 
